\title{
Portomesenteric Venous Gas and Pneumatosis Intestinalis
}

\author{
Norihiko Matsumura ${ }^{1}$, Takatomi Fujimoto ${ }^{2}$, Eiji Takase $^{2}$, Rina Hirohashi ${ }^{3}$ and Satoru Kitano ${ }^{3}$ \\ Key words: Portomesenteric venous gas, Pneumatosis intestinalis, CT scan, Intestinal ischemia
}

\author{
(DOI: 10.2169/internalmedicine.45.1672)
}
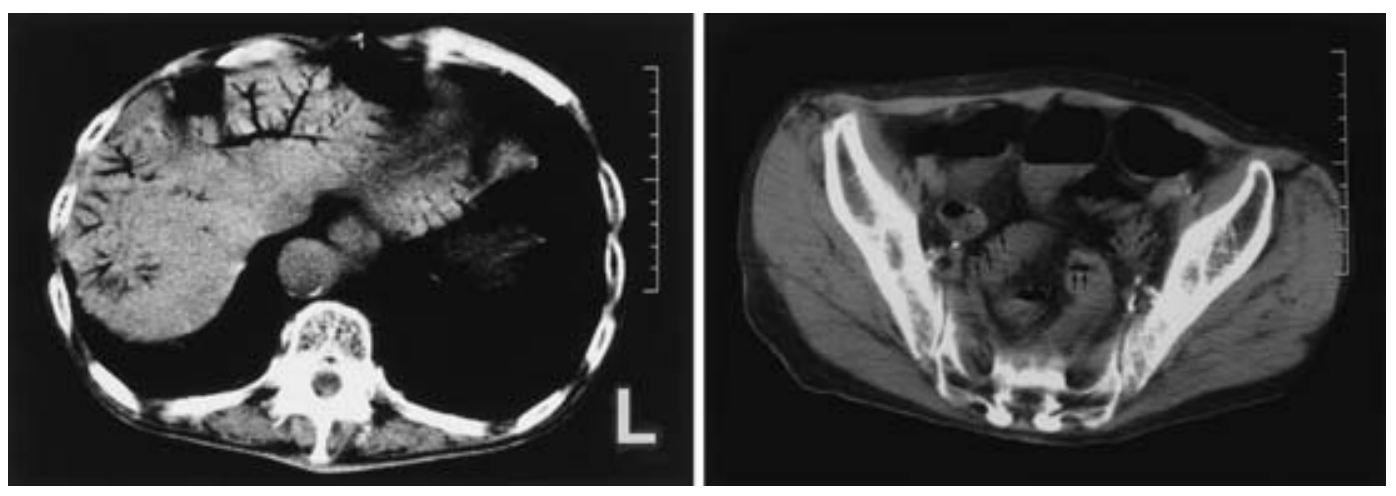

Figure 1. A computed tomographic (CT) scan of the abdomen showing: left, more extensive gas collection with a branching pattern in the intrahepatic portal veins, and right, bubble-like pneumatosis (black arrows) in the small intestines.

A 74-year-old man presented to the emergency room with diarrhea and abdominal pain of 3 days' duration. The abdomen was distended and diffusely tender without rebound, guarding, or masses. Laboratory data showed an inflammatory change and renal dysfunction with metabolic acidosis. Despite emergency hemodialysis and antimicrobial therapy, the patient became septic and deteriorated rapidly, and died the following day. A computed tomographic (CT) scan of the abdomen showed gas within the intrahepatic portal vein and gas collections in the small bowel wall (pneumatosis intestinalis) (Fig. 1).

The clinical course raises the possibility that he died of acute intestinal ischemia. Both CT findings of portomesenteric venous gas and pneumatosis intestinalis are highly associated with transmural bowel infarction (1). Liebman et al postulated that hepatic portal venous gas results from either transmural migration of gas from the intestinal lumen or from gasproducing enteric organisms within the portal venous system (2).

\section{References}

1. Wiesner W, Mortele KJ, Glickman JN, Ji H, Ros PR. Pneumatosis intestinalis and portomesenteric venous gas in intestinal ischemia: Correlation of CT findings with severity of ischemia and clinical outcome. Am J Roentgenol 177: 1319-1323, 2001.

2. Liebman PR, Patten MT, Manny J, Benfield JR, Hechtman HB. Hepatic-portal venous gas in adults: Etiology, pathophysiology and clinical significance. Ann Surg 187: 281-287, 1978.

(C) 2006 The Japanese Society of Internal Medicine

http://www.naika.or.jp/imindex.html

\footnotetext{
${ }^{1}$ Department of Nephrology, Saiseikai Suita Hospital, Suita, ${ }^{2}$ Department of Cardiology, Saiseikai Suita Hospital, Suita and ${ }^{3}$ Department of Radiology, Saiseikai Suita Hospital, Suita

Received for publication November 29, 2005; Accepted for publication December 22, 2005

Correspondence to Norihiko Matsumura, Department of Nephrology, Saiseikai Suita Hospital, 1-2 Kawazono-cho, Suita, Osaka 564-0013
} 